\title{
Calidad de agua de los tributarios de la Laguna del Sauce, Maldonado, Uruguay, en base al Îndice Diatomológico Pampeano (IDP)
}

\author{
Water quality of the tributaries \\ of the Sauce Lagoon, Maldonado-Uruguay, \\ based on the Pampean Diatom Index (PDI)
}

Recibido: 22/4/2019 $\rightarrow$ Aprobado: 29/5/2019 $\square_{\text {gferrari@latu.org.uy }}$

i̊ ferrari, graciela (1); KRÖGer, alejandra (1); torrendell, maría (2)

(1) Departamento de CAlidad de agua y eVAluación ambiental, latu, MONTEVIDEO, URUGUAY.

(2) Departamento de microbiología, LATU, montevideo, URUGuay.

\section{RESUMEN}

Se aplica por primera vez en Uruguay un bioindicador bentónico en monitoreos de calidad de agua en arroyos utilizando a las diatomeas. Estas microalgas son el grupo más diverso y suelen constituir el 80-90\% del perifiton. Son reconocidas en todo el mundo por su valor como indicadoras de eutrofización y polución orgánica, ya que responden rápidamente a los cambios ambientales y proveen de información sobre la integridad biótica de los ecosistemas acuáticos. En virtud de estas características se han desarrollado índices de calidad de agua con base en la presencia de determinadas especies en un sitio. El objetivo del trabajo fue determinar la calidad del agua que presentaban los afluentes de la cuenca de la Laguna del Sauce (Maldonado, Uruguay) en base a la aplicación del Índice Diatomológico Pampeano (IDP). Para ello se analizó el perifiton en 11 sitios de cinco tributarios del Sistema Laguna del Sauce cada dos meses, desde julio de 2015 a diciembre de 2016. De las 238 especies determinadas, 44 tuvieron una abundancia relativa mayor del 4\%, y de 26 se contaba con el valor indicador para el Índice Pampeano de Diatomeas (Idpi). Según el análisis de correspondencia, las especies se ordenaron en función de su relación con el nitrógeno total, el fósforo total y el fosfato. Se observó que los tributarios presentaron mala calidad del agua y que hacia la salida de la Laguna, en Represa y arroyo Potrero, la calidad fue aceptable. Los programas de monitoreo de calidad de agua podrían complementarse con la incorporación de esta herramienta y sería de gran utilidad en el asesoramiento del estado trófico de los cuerpos de aguas lóticos. PALABRAS CLAVE: diatomeas perifíticas, eutrofización, bioindicador. 


\section{ABSTRACT}

A benthic bioindicator is applied for the first time in water quality monitoring in streams in Uruguay using diatoms. These microalgae are the most diverse group and usually constitute $80-90 \%$ of periphyton. They are recognized throughout the world for their value as indicators of eutrophication and organic pollution because they respond quickly to environmental changes and provide information on the biotic integrity of aquatic ecosystems. Thanks to these characteristics, water quality indexes have been developed based on the presence of certain species in a site. The purpose of this work was to determine which water quality is present in the tributaries of the Sauce Lagoon basin (Maldonado, Uruguay) based on the application of the Pampean Diatom index (IDP). To achieve this, the periphyton in 11 sites of five tributaries of the Sauce Lagoon System was analyzed every two months from July 2015 to December 2016. Within the 238 species determined, 44 had a relative abundance greater than $4 \%$, and of 26 of them, information on the indicator value for the Pampean Diatom Index (Idpi) was available. According to the correspondence analysis, the species were ordered by total nitrogen, total phosphorus and phosphate. The tributaries presented poor quality of water. However, towards the lagoon drain in Represa site and Potrero stream, water quality improved and was considered as acceptable. The water quality monitoring programs could be complemented with the incorporation of this tool and it would be very useful in the assessment of the trophic state of the lotic waters.

KEYWORDS: periphytic diatoms, eutrophication, biological indicators.

\section{INTRODUCCIÓN}

La influencia del hombre en el medioambiente por el aumento de la densidad poblacional y la actividad industrial y agrícola impacta los ecosistemas acuáticos (Duarte, et al., 2006). El aumento progresivo del proceso de eutrofización a causa del enriquecimiento orgánico, fertilizantes y residuos de metales pesados y agroquímicos afecta la calidad de los cuerpos de agua, generando interferencias negativas en el suministro de agua potable y en los múltiples servicios ecosistémicos acuáticos (Carpenter, 2011). Con el fin de realizar evaluaciones ambientales, se han desarrollado métodos fisicoquímicos y biológicos que determinan el grado de impacto en los ecosistemas acuáticos (Unión Europea, 2000; Lobo y Callegaro, 2000; Springer, 2010). Para ello, se establecieron metodologías básicas para el establecimiento del estado ecológico de lagos y ríos y se realizaron los protocolos de muestreo y análisis de las comunidades biológicas a monitorear (Barbour, et al., 1999; Asociación Española de Normalización y Certificación, 2004; Cambra, et al., 2005; Confederación Hidrográfica del Ebro, 2007; Blanco, et al., 2010).

Los organismos indicadores más utilizados para el biomonitoreo son los organismos sésiles de un ecosistema. Por ello, los macroinvertebrados y las diatomeas bentónicas han sido las comunidades más estudiadas. Las comunidades de diatomeas responden rápidamente a los cambios ambientales y pueden proveer información sobre la integridad biótica de los ecosistemas acuáticos, definida como la capacidad que tiene un sistema 
acuático para soportar y sostener una comunidad de organismos adaptada, integrada y equilibrada, teniendo una diversidad de especies comparable a la del hábitat natural de una región (Prygiel, et al., 1999; Prygiel y Coste, 2000; Li, et al., 2010).

Las diatomeas son un grupo de microalgas muy diverso y suelen constituir el 80-90\% del perifiton o biofilm, es decir, del total de organismo que crece adherido en un sustrato fijo, ya sea una planta, piedra o arena, en un ecosistema acuático. Son reconocidas en todo el mundo por su valor como indicadoras de eutrofización y polución orgánica (Kelly y Whitton, 1995; Gómez y Licursi, 2001; Lobo, et al., 2004a; Lobo, et al., 2004b; Ector y Rimet, 2005). En virtud de estas características se han desarrollado, en distintas regiones del mundo, índices de calidad de agua basados en diatomeas, como el índice biológico de diatomeas (IBD) (Descy y Coste, 1991; Coste, et al., 2008), el índice de sensibilidad a la contaminación específica (ISP) (Cemagref, 1982), el índice sapróbico (SI) (Sládecék, 1986), el índice de diatomeas genérico (GDI) (Coste y Ayphassorho, 1991; Lenoir y Coste, 1996), el índice de asociación de diatomeas de la contaminación orgánica (DAIPo) (Watanabe, et al., 1986) y el índice trófico de diatomeas (Kelly y Whitton, 1995). Incluso se desarrolló un software, OMNIDIA, que genera un total de 18 índices diatomológicos (LeCointe, et al., 1993).

El Índice Biológico de Diatomeas (IBD) es un método de uso habitual en Francia para la vigilancia de la calidad de los cursos de agua. Este índice se basa en una lista de 209 especies clave con diferentes sensibilidades a diferentes tipos de contaminación (Coste, et al., 2008). Desde 1997 se ha demostrado que estos índices son una herramienta en la evaluación de las condiciones de los ríos (Hill, et al., 2000; Hill, et al., 2001). En Estados Unidos, la Agencia de Protección Ambiental (EPA por su sigla en inglés) estableció protocolos que utilizan a las especies de diatomeas pertenecientes al perifiton como bioindicadoras en los monitoreos ambientales (Barbour, et al., 1999).

Hay relativamente pocos antecedentes del uso de las diatomeas como indicadores de cambios en la calidad del agua en América del Sur, en comparación con América del Norte y Europa. En Argentina, Gómez y Licursi (2001) desarrollaron el Índice Diatomológico Pampeano (IDP) para evaluar la calidad del agua de los ríos y arroyos en la región pampeana de Argentina, sobre la base de la sensibilidad de las diatomeas epipélicas al enriquecimiento orgánico y la eutrofización (Licursi y Gómez, 2003). Por otra parte, la validación de estos índices ha permitido su empleo en otras áreas, como la cuenca del río Monjolinho, que atraviesa la ciudad de São Carlos, en el estado de São Paulo, Brasil (Bere y Tundisi, 2012), y en ríos subtropicales de Río Grande do Sul, Brasil (Lobo, et al., 2010). A pesar de que la taxonomía de las diatomeas uruguayas se conoce bien y existe un relevamiento taxonómico exhaustivo de diatomeas bentónicas realizado en sistemas acuáticos nacionales, que incluye ríos grandes (Río Uruguay), medianos, pequeñas cañadas y arroyos, lagos, lagunas y el estuario del Río de la Plata (Metzeltin, et al., 2005), no se han utilizado los índices diatomológicos para evaluar la calidad de agua en los sistemas lóticos de Uruguay.

Laguna del Sauce (Maldonado, Uruguay) es uno de los principales reservorios costeros que abastece de agua potable a más del 95\% de la población fija y flotante del departamento de Maldonado. Este cuerpo de agua ha sido declarado como clase 1: aguas destinadas al abastecimiento de agua, según el Decreto 253/79 (Uruguay, 1979). 
El sistema Laguna del Sauce presenta un grado de eutrofización elevado, evidenciado por la recurrencia y persistencia de floraciones de cianobacterias (Rodríguez, et al., 2010; Mazzeo, et al., 2010; González-Madina, 2017). Para mitigar la eutrofización se generó un plan de acción y medidas de control de la degradación ambiental (MVOTMA, 2015).

El objetivo de este trabajo fue aplicar el IDP en los afluentes de la cuenca de una fuente de agua potable como Laguna del Sauce, contemplando la necesidad de contar con bioindicadores de la calidad de agua que muestren de manera rápida e integrada el estado trófico de un sitio y que permitan visualizar las principales entradas de nutrientes al sistema. La aplicación del IDP permite, además, contribuir a dar seguimiento al plan de acción y a las medidas de control en la gestión de este recurso hídrico. Este índice determina cinco niveles en la calidad del agua de los afluentes, lo que redundará en la gestión y la toma de medidas para el control de los vertidos puntuales y difusos, así como también en la evaluación de los efectos de la eutrofización en el sistema. En este trabajo también se analizaron las variables ambientales con las cuales fue construido y desarrollado el IDP para dar una mayor interpretación y validar su aplicación.

\section{MATERIALES Y MÉTODOS}

\section{Área de estudio}

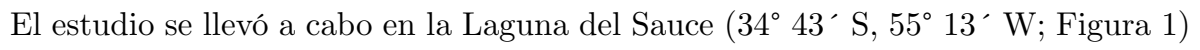
y sus tributarios (departamento de Maldonado, Uruguay). El sistema Laguna del Sauce está integrado por tres cuerpos de agua someros conectados, Laguna del Potrero (441 ha), Laguna de los Cisnes (205 ha) y Laguna del Sauce (4050 ha), que forman en su totalidad un espejo de agua de 4.666 ha. Este sistema presenta una profundidad media de 2,5 m y máxima de $5 \mathrm{~m}$ (Mazzeo, et al., 2010).

Los principales tributarios que desembocan en Laguna del Sauce son el arroyo Pan de Azúcar, que nace en la Sierra de las Ánimas y atraviesa la ciudad de Pan de Azúcar recorriendo aproximadamente $22 \mathrm{~km}$, y el arroyo Sauce, que nace en la Sierra Catedral y recorre aproximadamente $16 \mathrm{~km}$. Otros tributarios son las cañadas Pedregosa y Mallorquina y los arroyos Salto de Agua y Enjoy (Figura 1). Al sur, la Laguna tiene un desagüe natural en el Río de la Plata constituido por el arroyo del Potrero (Rodríguez, et al., 2010).

Los $426 \mathrm{~km}^{2}$ que comprenden la cuenca de Laguna del Sauce se distribuyen de la siguiente manera, según el uso del suelo: el 61,1\% corresponde a ganadería sustentada en campo natural, el 11,1\% a plantaciones forestales, el 9,6\% a monte nativo, el $6 \%$ al espejo de agua de la laguna, el 5,1\% a área agrícola, el 4,8\% a suelos pobres, mientras que el 2,2\% restante se distribuye en bañados y humedales, parques, centros poblados y área turística (Amorín y Larghero, 2017; Rodríguez, et al., 2010). Como se mencionó anteriormente, la Laguna del Sauce abastece a la gran mayoría de la población fija y flotante del departamento de Maldonado, constituyendo la segunda fuente de agua potable de Uruguay para un total de 140.000 habitantes, que aumentan a 400.000 en época estival (Méndez, et al., 2010). 
Desde el punto de vista histórico, cabe mencionar algunos hechos clave que pueden ayudar a explicar el estado actual de la calidad del agua de Laguna del Sauce. En 1947 la laguna fue represada para mantener el nivel del agua con fines aeronaúticos, lo que aumentó el espejo de agua y el tiempo de residencia, incrementando las tasas de retención de nutrientes y disminuyendo las tasas de pérdidas de biomasa algal fuera del sistema (Rodríguez, et al., 2010). Además, aumentaron los aportes de nutrientes desde la cuenca de drenaje, producto de un mayor desarrollo agrícola-ganadero, urbano y turístico. En consecuencia, esto determinaría una elevada carga externa y reserva interna de nutrientes (principalmente asociada al sedimento) capaz de sostener el crecimiento excesivo de productores primarios (microalgas o plantas acuáticas). Las floraciones microalgales, y en particular de cianobacterias, presentan importantes inconvenientes, ya que la presencia de estos organismos supone riesgo sanitario por su capacidad de producir toxinas, lo cual puede provocar trastornos en el suministro de agua potable, así como en las actividades productivas y/o recreativas. Por este motivo, el manejo adecuado de este fenómeno es un factor crítico (Rodríguez, et al., 2010).

\section{COLECTA DE MUESTRAS}

Los sitios de muestreo fueron seleccionados de acuerdo al monitoreo mensual de la red de tributarios que realiza la Unidad de Gestión Desconcentrada (OSE-UGD) en 11 sitios ubicados en afluentes de la laguna: (1) Mallorquina, (2) Pedregosa, (3) Sauce, (4) Salto de Agua AB, (5) Salto de Agua AA, (6) Pan de Azúcar AA, en puente de la Ruta 9, (7) Pan de Azúcar AB, (8) Vivero, (9) Enjoy (arroyo sin nombre oficial, pero tiene una propaganda del Hotel Enjoy), (10) Represa y (11) Potrero, que conecta con el Río de la Plata (Figura 1). Los muestreos se realizaron bimestralmente desde octubre de 2015 a setiembre de 2016 (octubre y diciembre en 2015 y febrero, abril y setiembre en 2016).

En cada sitio se tomaron medidas in situ con la sonda multiparámetro YSI 6920 $\mathrm{V} 2$, que incluye sensores de temperatura $\left({ }^{\circ} \mathrm{C}\right)$, conductividad $(\mu \mathrm{S} / \mathrm{cm})$, oxígeno disuelto $(\mathrm{mg} / \mathrm{l}), \mathrm{pH}$ y turbiedad (UNT). Las variables analizadas fueron las mismas que sirvieron para construir el IDP, realizadas en los trabajos de Gómez y Licursi (2001). Se tomaron muestras de agua en forma directa para análisis de nitrógeno total (NT) basado en ISO 11905-2 (International Organization for Standardization, 1997) y fósforo total (PT) según APHA 4500P (American Public Health Association, et al., 2017), QuikChem method 31-115-01-3-D (Lachat Instruments, 2007), y Clorofila a según ISO 10260 (International Organization for Standardization, 1992). En el muestreo de setiembre de 2016 se agregaron análisis de amonio (NH4), basados en APHA 4500 NH3-D (American Public Health Association, et al., 2017), demanda biológica de oxígeno (DBO), basado en ISO 5815-2 (International Organization for Standardization, 2003), demanda química de oxígeno (DQO), según ISO 15705 (International Organization for Standardization, 2002), nitritos, basado en ISO 10304-1 (International Organization for Standardization, 2007), y nitratos, según ISO 10304-1 (International Organization for Standardization, 2007), sólidos suspendidos totales, basado en APHA 2540 D (American Public Health Association, 
et al., 2017), y carbono orgánico total, según ISO 8245 (International Organization for Standardization, 1999). Los datos de fosfato fueron proporcionados por OSEUGD para cada sitio y fecha de muestreo.

Las diatomeas epilíticas crecen unidas a rocas en la zona fótica de arroyo, por lo que el muestreo consistió en el raspado de rocas sumergidas hasta $20 \mathrm{~cm}$ de profundidad siguiendo la metodología sugerida por Kelly et al. (1998) y Nora Gómez (Comunicación personal), además de los protocolos de la comunidad europea (Asociación Española de Normalización y Certificación, 2004) y de la EPA (Barbour, et al., 1999). En cada sitio se obtuvo una muestra de perifiton raspando con un cepillo dental y utilizando un cuadrante de goma eva de $3 \times 3 \mathrm{~cm}$ de unas 10 rocas elegidas al azar situadas en diferentes posibles microhábitats (con/sin vegetación, ambas riberas).

Las muestras para análisis taxonómico fueron obtenidas por duplicado; una réplica se transportó fresca y en oscuridad para ser procesada al otro día, y la otra fue fijada in situ con formol neutro al 4\%. Para los análisis de clorofila del perifiton se extrajo otra muestra raspando una superficie de $9 \times 9 \mathrm{~cm}$ que se filtró en campo. El filtro obtenido se congeló hasta su análisis en laboratorio. En los sitios donde no se encontraron rocas, tales como $\mathrm{A}^{\underline{0}}$ Mallorquina, Pan de Azúcar AB y Vivero, se utilizaron sustratos artificiales de acero inoxidable construidos con 24 caras de $9 \times 9 \mathrm{~cm}$ cada una, colocados en un muestreo previo, en julio de 2015 (Figura 2).

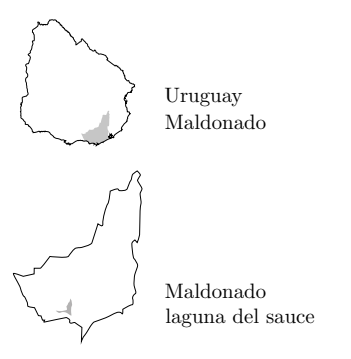

- 1 Mallorquina

- 2 Pedragosa

- 3 Sauce

- 4 Salto de Agua AB

- 5 Salto de Agua AA

- 6 Pan de Azúcar AA

- 7 Pan de Azúcar AB

- 8 Vivero

- 9 Enjoy

10 Represa

11 Potrero

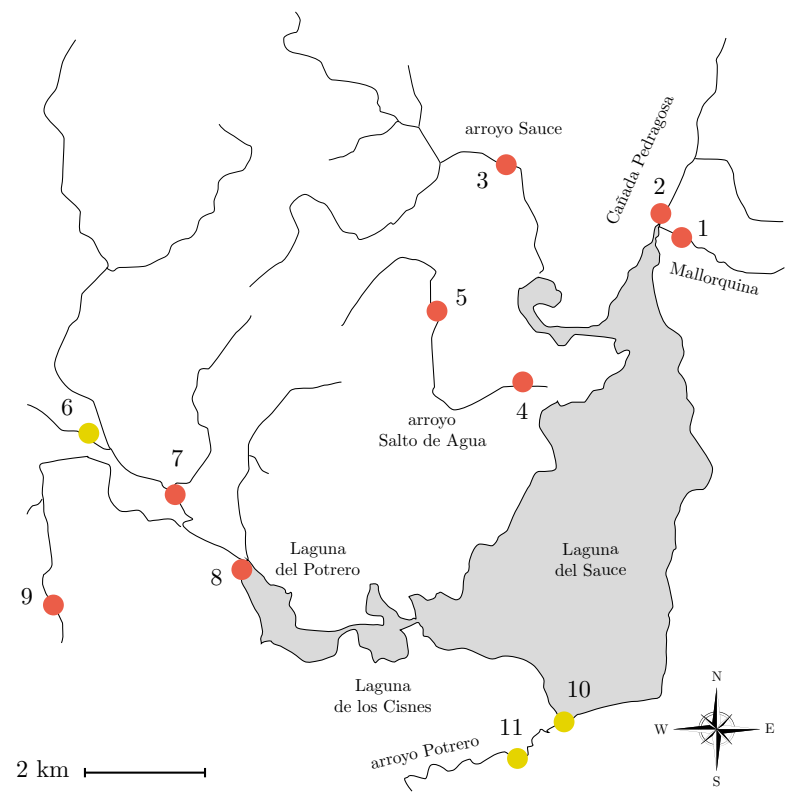

Figura 1. Sistema de lagunas de la cuenca de Laguna del Sauce: Laguna de Potrero, Laguna del Cisne y Laguna del Sauce. Localización de los sitios de muestreo: (1) Mallorquina, (2) Pedregosa, (3) Sauce, (4) Salto de Agua AB, (5) Salto de Agua AA, (6) Pan de Azúcar AA, en un puente de la Ruta 9, (7) Pan de Azúcar AB, (8) Vivero, (9) Enjoy, (10) Represa y (11) Potrero. Índice de colores de acuerdo a calidad de agua inferida a partir del IDP (promedio de los cinco muestreos, ver Figura 6). 


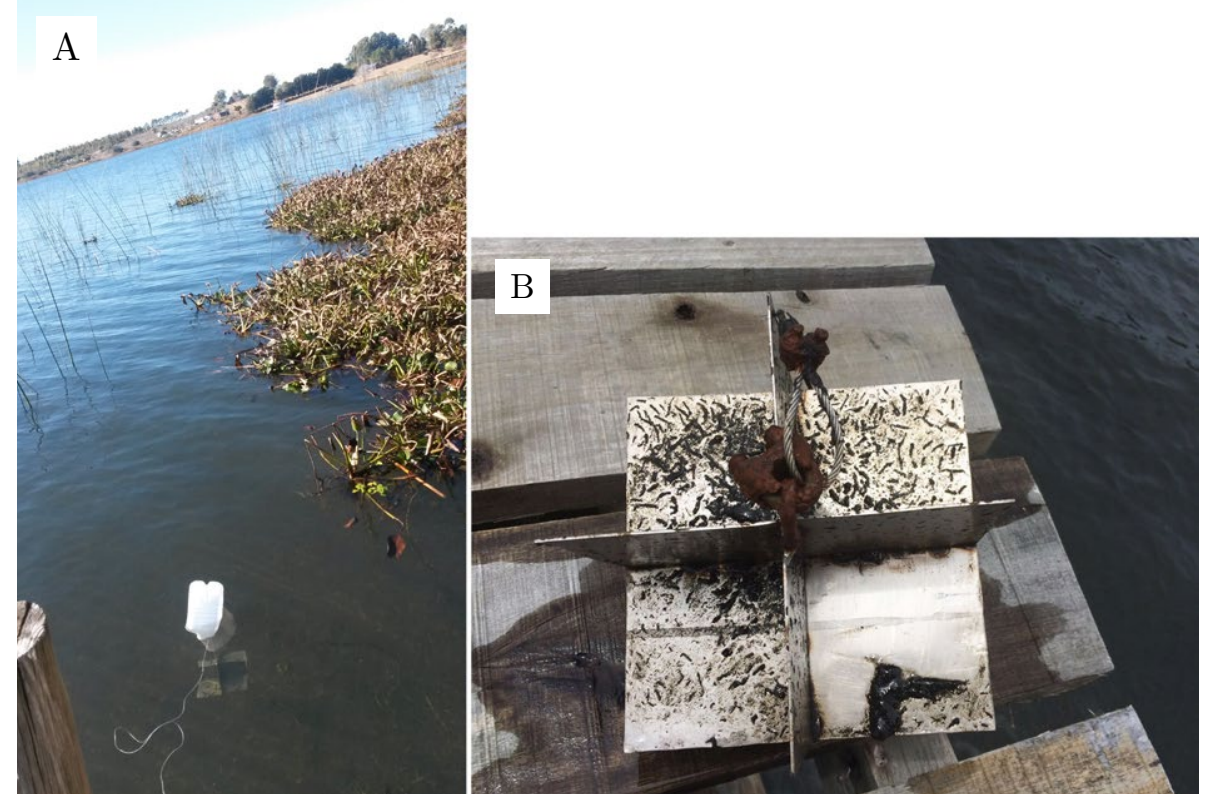

Figura 2. A) Sustrato puesto en la laguna y B) raspado de sustrato artificial.

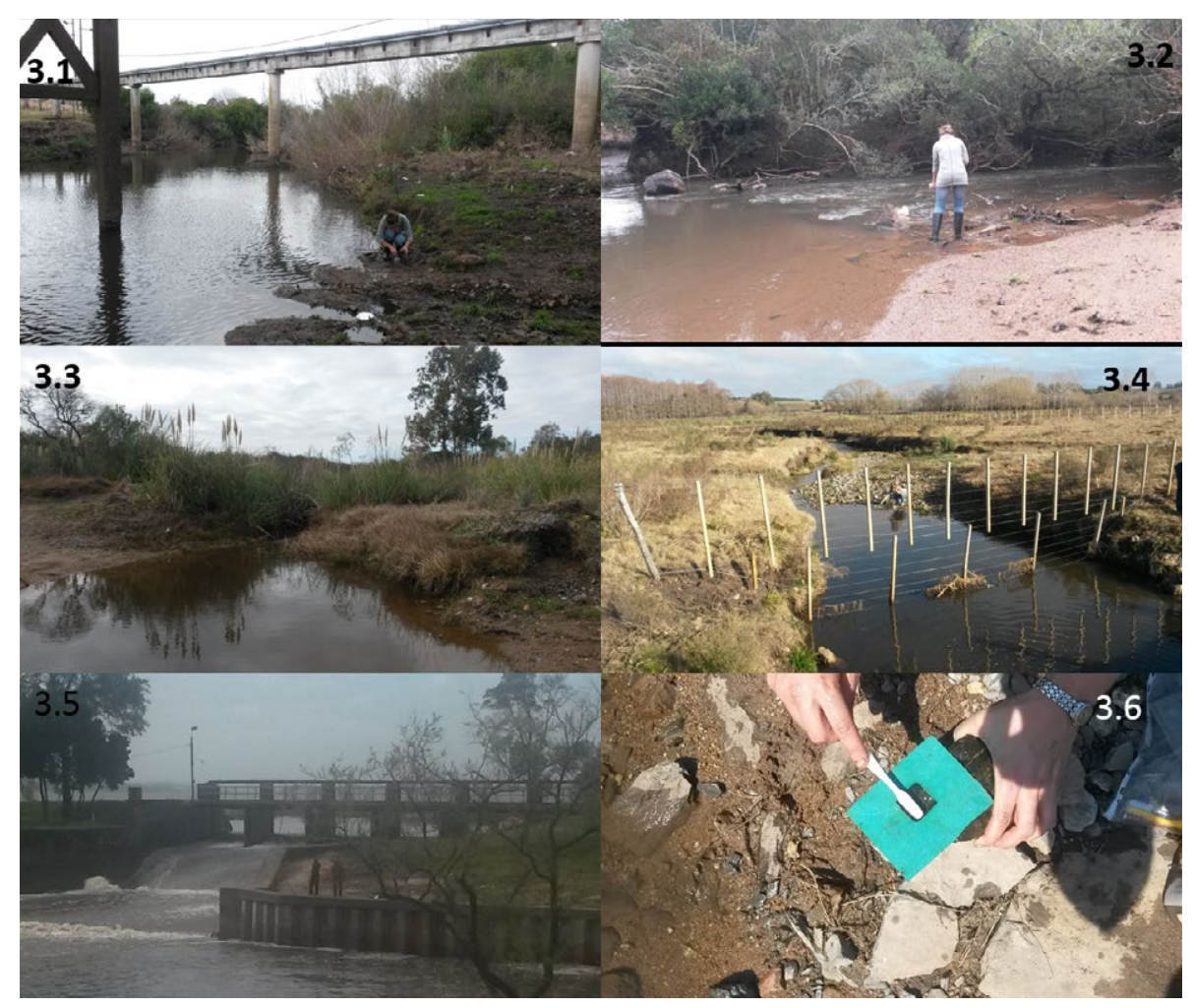

Figura 3. 3.1: Pan de Azúcar (sitio 6- PA AA), 3.2: Pan de Azúcar (sitio 7-AB), 3.3: Salto de agua (sitio 4-AB), 3.4: Pedragosa, 3.5: Represa (setiembre 2016), 3.6: raspado de rocas.Análisis cualitativo y cuantitativo de las poblaciones de diatomeas 
En el laboratorio las muestras se oxidaron con peróxido de hidrógeno al $30 \%$ siguiendo metodología sugerida por Battarbee (1986). Se transfirió $5 \mathrm{~mL}$ de cada muestra a un tubo de ensayo, se le agregó $5 \mathrm{~mL}$ de peróxido para tener una proporción 1:1, y se calentó en una placa calefactora durante 10 minutos o hasta observar el cambio de color del líquido. Luego se lavó con agua destilada mediante tres a cinco centrifugaciones sucesivas. Se preservó cada muestra con formaldehído al 4\% y se realizaron preparados permanentes con la resina sintética Naphrax@. Para la identificación taxonómica se utilizó un microscopio óptico Olympus BX53, equipado con óptica DIC, que tiene incorporada una cámara Infinitive I con Image Pro Plus software para análisis de imagen. Las observaciones se realizaron bajo una magnificación de 1000x. Asimismo, se realizaron observaciones con un microscopio electrónico de barrido Zeiss Supra 40, del Centro de Microscopías Avanzadas de la Universidad de Buenos Aires. Para la identificación de las especies de diatomeas se utilizó bibliografía de referencia taxonómica como la colección de diatomeas de Europa (Krammer, 2000, 2002 y 2003; Lange-Bertalot, 2001 y 2011; Levkov, 2009, 2013 y 2016) y de Estados Unidos (EPA, [s.d.]), y regionales, como Sar et al. (2009) y Metzeltin et al. (2005), entre otros. Los análisis cuantitativos fueron realizados en los preparados permanentes y los conteos fluctuaron en el entorno de 400 valvas, número suficiente para obtener una buena representación de las especies que tienen al menos abundancia relativa superior al 5\% (Licursi y Gómez, 2003).

\section{Análisis de datos}

Para determinar el conjunto de variables ambientales que contribuyen a explicar la variabilidad de la abundancia de diatomeas, se realizó un Análisis de Correspondencia Canónica (CCA por su sigla en inglés) con el método unimodal de ordenación, dado que este análisis es válido para cualquier tamaño del gradiente ambiental (Legendre y Legendre, 2012). Se utilizó la matriz de especies con abundancia relativa mayor al $4 \%$ transformada $(\log x+1)$. Para la matriz ambiental se utilizaron los datos medidos in situ y los obtenidos de los análisis químicos (NT, PT, PO4, clorofila) como variables explicativas que fueron normalizadas. La significancia de las contribuciones de las variables ambientales en la ordenación de las especies se probó mediante la prueba de permutaciones de Monte Carlo $(\mathrm{p}<0,05)$. La significancia de los coeficientes de correlación canónica de cada variable ambiental para los ejes 1 y 2 se evaluó mediante una aproximación del estadístico t-Student (ter Braak, 1986) siguiendo las rutinas del software libre MVSP (Multivariate Statistical Package). El nitrito, la DQO y el amonio fueron eliminados del análisis debido a que su concentración en todos los sitios fue menor al límite de detección, así como la temperatura (para no considerar la variación estacional). Para evaluar el rol de cada especie y su contribución al sitio se calcularon los coeficientes de similitud entre muestras y la similitud/disimilitud promedio entre todos los pares de muestras de cada grupo, aplicando el análisis de porcentajes de similaridad mediante la rutina SIMPER del PRIMER. A las 
especies que más contribuyen a la similitud promedio dentro de un sitio se las consideró como típicas (Clarke y Warwick, 2001). Con esta matriz de especies/ sitio también se realizó un análisis de varianza ANOSIM para comparar sitios mediante matrices de similitud de Bray-Curtis. Estos análisis fueron realizados con el software PRIMER v6 (Clarke y Gorley, 2006).

El IDP de cada sitio fue calculado siguiendo a Gómez y Licursi (2001) y Licursi y Gómez (2003). La abundancia relativa de cada taxón fue multiplicada por el valor de ponderación de la especie: «índice específico"(Iidp). La sumatoria del valor de todas las especies ponderadas para cada muestra determinó el IDP del sitio, tal como indican las autoras. El IDP genera cinco clases de calidad de agua (0-4) que siguen la clasificación de la Tabla 1. Para la coloración de los sitios de la Figura 1, el IDP del sitio se tomó como el promedio de los valores del IDP de los cinco muestreos realizados.

TABLA 1. Valor del IDP, categoría de calidad de agua y su correspondiente código en color. Tomado de Licursi y Gómez (2003).

\begin{tabular}{|c|c|c|}
\hline Valor IDP & Calidad de agua & Código de color \\
\hline $0-0,5$ & Muy buena & \\
\hline$>0,5-1,5$ & Buena & \\
\hline$>1,5-2$ & Aceptable & \\
\hline$>2-3$ & Mala & \\
\hline$>3-4$ & Muy mala & \\
\hline
\end{tabular}

\section{RESULTADOS}

\section{Variables ambientales}

En la Tabla 2 se muestran los promedios de las variables ambientales en cada sitio de todos los muestreos realizados $(n=5)$ y los datos puntuales tomados en el muestreo de setiembre de 2016.

Se observó que los mayores aportes de nutrientes al Sistema Laguna del Sauce provienen del norte (Mallorquina y Pedragosa) y del arroyo Enjoy, donde se registraron las mayores concentraciones de nitrógeno total, fósforo total y fosfato. $\mathrm{El}$ arroyo Mallorquina presentó un pico de PT de $1050 \mu \mathrm{g} / \mathrm{l}$ en abril. Este sitio se caracteriza por tener plantas enraizadas, que favorecen la transparencia del agua, por lo que la turbiedad fue baja (1 UNT) en todos los muestreos.

La profundidad de los arroyos en los sitios de muestreo no superó los $50 \mathrm{~cm}$, con excepción del sitio Vivero $(1 \mathrm{~m})$. No se observó un gradiente ambiental en los arroyos que tienen más de un sitio de muestreo en su curso de agua, por ejemplo, Salto de Agua y Pan de Azúcar. 
TABLA 2. Los promedios y desvío de las variables ambientales en cada sitio de muestreo standard $(\mathrm{n}=5)$, máximos y mínimos de todos los datos. Octubre 2015-setiembre 2016. (*) Datos tomados en setiembre de 2016. (**) Datos proporcionados por OSE-UGD. ND: No Detectado, SR: sin registro. T: temperatura, Cond: conductividad, UNT: turbiedad, OD: oxígeno disuelto, NT: Nitrógeno total, PT: fósforo total, Chl a: Clorofila $a$, NH4: amonio, DBO: demanda biológica de oxígenos, DQO: demanda bioquímica de oxígeno, NO2: nitrito, NO3: nitrato, SST: sólidos suspendidos totales y PO4: fosfato.

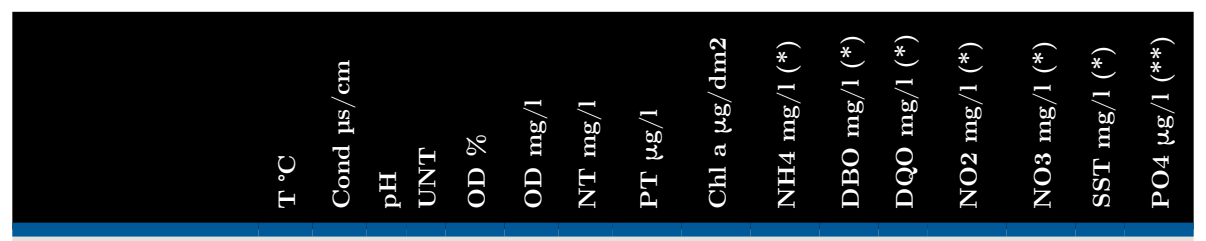

\begin{tabular}{|c|c|c|c|c|c|c|c|c|c|c|c|c|c|c|c|}
\hline Mallorquina (media) & 17,5 & 532 & 7,3 & 3 & 40,0 & 3,0 & 1,3 & 386,0 & 56,0 & 0,05 & 2,6 & $<14<0,046$ & 0,03 & 3,8 & 303 \\
\hline Mallorquina (desvest) & 2,5 & 71 & 0,8 & 4 & 37,6 & 2,3 & 1,0 & 454,8 & 57,8 & & & & & & \\
\hline Pedragosa (media) & 24,7 & 616 & 7,9 & 21 & 102,0 & 11,0 & 1,1 & 113,0 & 60,7 & 0,04 & 1,45 & $\mathrm{ND}<0,046$ & 0,01 & 3,4 & 53 \\
\hline Pedragosa (desvest) & 5,9 & 147 & 0,8 & 37 & 43,9 & 5,7 & 1,0 & 69,2 & 48,7 & & & & & & \\
\hline Sauce (media) & 20,2 & 268 & 7,4 & 4 & 74,0 & 7,0 & 0,7 & 146,0 & 38,4 & 0,03 & 1,5 & $\mathrm{ND}<0,046$ & 0,04 & 1 & 98 \\
\hline Sauce (desvest) & 3,5 & 63 & 0,3 & 2 & 18,4 & 2,0 & 0,1 & 127,8 & 43,6 & & & & & & \\
\hline $\begin{array}{l}\text { Salto de agua } \mathrm{AB} \\
\text { (media) }\end{array}$ & 19,2 & 439 & 7,5 & 8 & 86,0 & 13,0 & 0,9 & 119,0 & 51,3 & 0,04 & 1,8 & $<14<0,046$ & 0,07 & 4 & 190 \\
\hline $\begin{array}{l}\text { Salto de agua } \mathrm{AB} \\
\text { (desvest) }\end{array}$ & 4,4 & 221 & 0,3 & 10 & 21,8 & 11,7 & 0,1 & 21,1 & 56,1 & & & & & & \\
\hline Salto de Agua AA $(*)$ & 17 & 180 & 7,5 & SR & 94,0 & 9,0 & 0,7 & 118,0 & 15,0 & 0,04 & 1,6 & $\mathrm{ND}<0,046$ & 0,06 & 6,8 & 260 \\
\hline Pan de Azúcar AA $\left(^{*}\right)$ & 13,9 & 249 & 7,9 & SR & 90,0 & 9,0 & 0,3 & 85,0 & 14,6 & 0,04 & 0,9 & $\mathrm{ND}<0,046$ & 0,06 & 3,6 & 68 \\
\hline $\begin{array}{l}\text { Pan de Azúcar AB } \\
\text { (media) }\end{array}$ & 18,7 & 266 & 7,5 & 2 & 74,0 & 7,0 & 0,5 & 113,0 & 56,3 & 0 & 0,17 & $<14<0,046$ & 0,07 & 3,6 & 83 \\
\hline $\begin{array}{l}\text { Pan de Azúcar AB } \\
\text { (desvest) }\end{array}$ & 2,8 & 67 & 0,4 & 1 & 12,8 & 1,4 & 0,2 & 61,8 & 38,1 & & & & & & \\
\hline Vivero (media) & 21,6 & 251 & 7,0 & 2 & 23,0 & 15,0 & 0,7 & 142,0 & 138,0 & 0 & 0,17 & $14<0,046$ & 0,04 & 78 & 65 \\
\hline Vivero (desvest) & 3,8 & 36 & 0,6 & 1 & 15,5 & 28,2 & 0,3 & 42,8 & 59,4 & & & & & & \\
\hline Enjoy $(*)$ & 15,5 & 808 & 8,1 & SR & 85,0 & 8,0 & 1,3 & 475,0 & 74,8 & 0 & 2,2 & $<14<0,046$ & 0,21 & 12 & 5690 \\
\hline Represa (media) & 22,7 & 163 & 7,3 & 10 & 60,0 & 5,0 & 1,1 & 89,0 & 94,6 & 0 & 0,8 & $49<0,046$ & 0,24 & 8,8 & 105 \\
\hline Represa (desvest) & 3,9 & 27 & 1,0 & 9 & 25,9 & 2,4 & 0,6 & 38,6 & 41,7 & & & & & & \\
\hline Potrero $(*)$ & 16,6 & 118 & 8,1 & $\mathrm{SR}$ & 99,0 & 10,0 & 0,9 & 145,0 & 41,1 & 0,07 & 1,3 & $<14<0,046$ & 0,26 & 6,4 & 280 \\
\hline Máx & 33,6 & 808 & 9,2 & 75 & 137,6 & 65,4 & 2,9 & 1050,0 & 168,0 & 0,07 & 2,6 & ND & 0,26 & 78 & 7130 \\
\hline Mín & 13,9 & 118 & 6,1 & ND & 5,2 & 0,7 & 0,3 & 34,0 & 0,0 & 0,003 & 0,167 & ND & 0,01 & 1 & 30 \\
\hline
\end{tabular}




\section{Comunidad de diatomeas}

De un total de 238 especies determinadas, 44 tuvieron una abundancia relativa mayor del $4 \%$ en todos los muestreos. En la Tabla 3 se presentan los taxones con su abundancia relativa, código e índice específico (Iidp). De las 44 especies seleccionadas, el 60\% cuenta con la ponderación del índice específico. En la Figura 4 se incluyen imágenes de especies representativas para cada Iidp.

De acuerdo al índice, fue posible ponderar un promedio de $70 \%$ de la abundancia de especies de cada muestra. En el caso del sitio Vivero (octubre), el $80 \%$ de las valvas no tenían ponderación, ya que Planotidium biporum no cuenta con Iidp, y es la especie dominante. Sin embargo, en $\mathrm{A}^{\mathrm{o}}$ Sauce, en abril, el $98 \%$ de las valvas tenían ponderación, ya que la especie dominante fue Cocconeis placentula.

TABLA 3. Especies frecuentes, con máxima abundancia relativa mayor al $4 \%$ en todo el período. Índice específico (Iidp), tomado de Licursi y Gómez (2003).

\begin{tabular}{|c|c|c|c|}
\hline Código & Especie & $\begin{array}{c}\text { Îndice } \\
\text { específico } \\
\text { Iidp }\end{array}$ & $\begin{array}{l}\text { Máxima } \\
\text { abundancia } \\
\text { relativa }\end{array}$ \\
\hline AEXG & Achnanthes exigua Grunow in Cleve y Grunow & 1 & 19,1 \\
\hline AАTO & $\begin{array}{l}\text { Achnanthidium atomus (Hustedt) Monnier, } \\
\text { Lange-Bertalot y Ector }\end{array}$ & & 20,3 \\
\hline ADMI & Achnanthidium minutissimum (Kützing) Czarnecki & 1,25 & 59,5 \\
\hline APED & Amphora pediculus (Kützing) Grunow & & 46,2 \\
\hline BPAX & Bacillaria paxilifer (Müller) Marsson & 1,75 & 16,5 \\
\hline CPLE & Cocconeis euglypta Ehrenberg & 2,25 & 12,1 \\
\hline CLIN & Cocconeis lineata Ehrenberg & & 9,2 \\
\hline CPLA & Cocconeis placentula Ehrenberg & 2 & 96,6 \\
\hline CMEN & Cyclotella meneghiniana Kützing & & 9,1 \\
\hline ESPR & Encyonema sprechmanii Metzeltin et al. & & 38,7 \\
\hline ESUB & Craticula subminuscula Wetzel y.Ector & 3,75 & 21,2 \\
\hline EMIN & Sellaphora nigri (De Notaris) Wetzel y.Ector & & 7,6 \\
\hline EADN & Epithemia adnata (Kützing) Brébisson & 1,25 & 15,1 \\
\hline ESOR & Epithemia sorex Kützing & 1,75 & 78,2 \\
\hline FACU & Ulnaria acus (Kützing) Aboal & & 11,7 \\
\hline FCAP & Fragilaria capucina Desmazières & 0,5 & 84,7 \\
\hline FGOU & $\begin{array}{l}\text { Ulnaria goulardii (Brébisson ex Cleve y Grunow) } \\
\text { Lange-Bertalot }\end{array}$ & & 7,6 \\
\hline FRUM & Fragilaria rumpens (Kützing) Carlson & & 11,3 \\
\hline
\end{tabular}




\begin{tabular}{|c|c|c|c|}
\hline Código & Especie & $\begin{array}{l}\text { Îndice } \\
\text { específico } \\
\text { Iidp }\end{array}$ & $\begin{array}{c}\text { Máxima } \\
\text { abundancia } \\
\text { relativa }\end{array}$ \\
\hline GPUN & $\begin{array}{l}\text { Geissleria punctifera (Hustedt) Metzeltin, } \\
\text { Lange-Bertalot y García-Rodríguez }\end{array}$ & & 8,3 \\
\hline GPUM & $\begin{array}{l}\text { Gomphonema pumilum (Grunow) Reichardt } \\
\text { y Lange-Bertalot }\end{array}$ & & 38,3 \\
\hline GPAR & Gomphonema parvulum (Kützing) Kützing & 3,25 & 29,9 \\
\hline HCOP & $\begin{array}{l}\text { Amphora copulata (Kützing) Schoeman } \\
\text { y Archibald }\end{array}$ & 2,5 & 17,5 \\
\hline KNIT & $\begin{array}{l}\text { Karayevia nitidiformis (Lange-Bertalot) } \\
\text { Bukhtiyarova }\end{array}$ & & 7,7 \\
\hline LHUN & Lemnicola hungarica (Grunow) Round y Basson & 2,5 & 8,4 \\
\hline LGEO & $\begin{array}{l}\text { Luticola goeppertiana (Bleisch) Mann ex Rarick, } \\
\text { Wu, Lee y Edlund }\end{array}$ & 3,75 & 8,3 \\
\hline LMUT & Luticola mutica (Kützing) Mann & 3 & 5,7 \\
\hline MVAR & Melosira varians Agardh & 2 & 45,5 \\
\hline NERI & Navicula erifuga Lange-Bertalot & 1,75 & 15,9 \\
\hline NKUS & Navicula kuseliana Lange-Bertalot y Rumrich & & 7,7 \\
\hline NCAP & Navicula capitatoradiata Germain & 1,25 & 31,5 \\
\hline NGRE & Navicula gregaria Donkin & 2,75 & 16,1 \\
\hline NSCHR & Navicula schroeteri Meister & & 33,5 \\
\hline NCLA & Nitzschia clausii Hantzsch & & 30,2 \\
\hline NACI & Nitzschia acicularis (Kützing) Smith & 3,75 & 6,3 \\
\hline NAMP & Nitzschia amphibia Grunow & 2,5 & 16,9 \\
\hline NFIL & Nitzschia filiformis (Smith) Van Heurck & & 4,6 \\
\hline NLIN & Nitzschia linearis Smith & 2,5 & 10,9 \\
\hline NSIG & Nitzschia sigmoidea (Nitzsch) Smith & 3 & 4,0 \\
\hline NPAL & Nitzschia palea (Kützing) Smith & 3,75 & 55,2 \\
\hline PACRO & Pinnularia acrosphaeria Smith & & 9,5 \\
\hline PFRE & Planothidium frequentissimum Lange-Bertalot & & 47,5 \\
\hline PBIP & $\begin{array}{l}\text { Planothidium biporomum (Hohn y Hellerman) } \\
\text { Lange-Bertalot }\end{array}$ & & 70,9 \\
\hline RSIN & Reimeria sinuata (Gregory) Kociolek y Stoermer & & 4,7 \\
\hline RABR & $\begin{array}{l}\text { Rhoicosphenia abbreviata (Agardh) Lange- } \\
\text { Bertalot }\end{array}$ & 1,5 & 87,0 \\
\hline SDEN & $\begin{array}{l}\text { Sellaphora densistriata (Lange-Bertalot y } \\
\text { Metzeltin) Lange-Bertalot y Metzeltin }\end{array}$ & & 6,4 \\
\hline THUN & Tryblionella hungarica (Grunow) Frenguelli & 2,75 & 10,6 \\
\hline
\end{tabular}




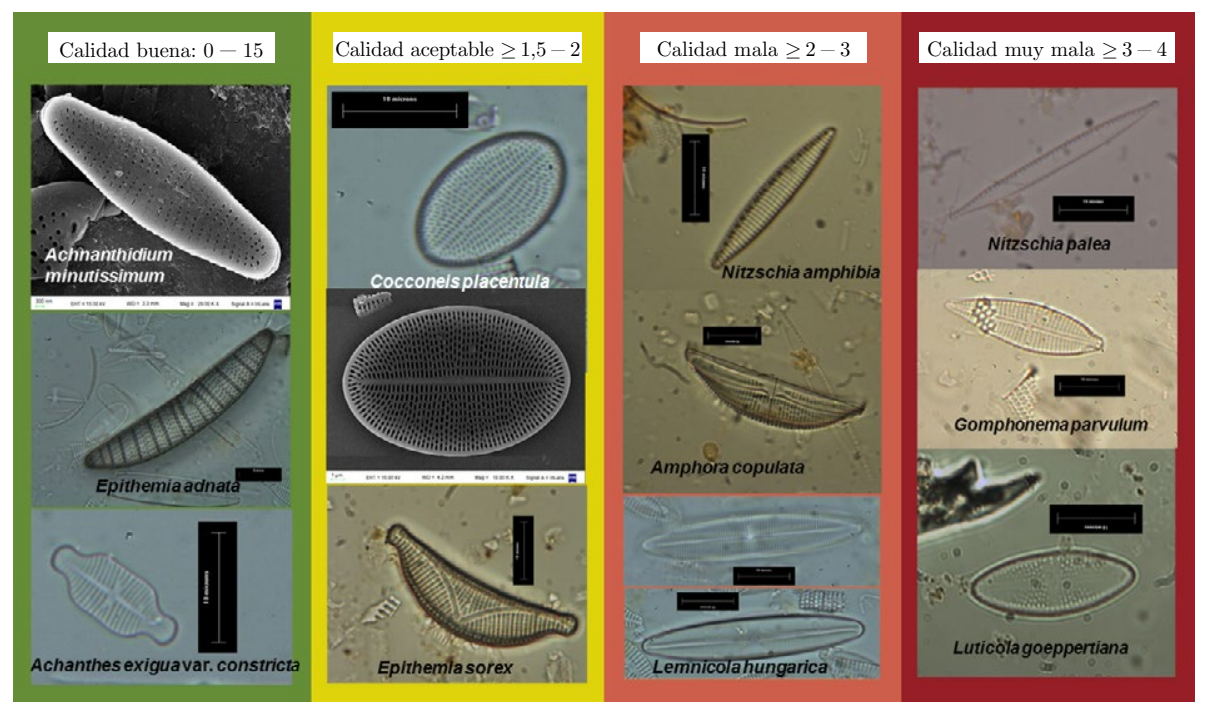

Figura 4. Especies representativas de cada categoría de calidad de agua según el valor de Iidp.

Cocconeis placentula, Rhoicosphenia abbreviata, Fragilaria capucina, Ephitemia sorex y Planothidium biporomum fueron las especies con mayor abundancia relativa (>70\%). Cada sitio presentó especies típicas (Tabla 4) y el resultado del análisis de similaridad ANOSIM mostró diferencias significativas entre los sitios $(\mathrm{R}$ global $=0,255 ; \mathrm{p}=0,01)$.

TABLA 4. Resultados del análisis de similaridad SIMPER: especies típicas en cada sitio y porcentaje de contribución de cada especie. Promedio de la abundancia, promedio de similaridad, porcentaje de contribución de la especie y porcentaje acumulado para cada sitio de muestreo.

\begin{tabular}{|c|c|c|c|c|c|}
\hline Sitio & Especies & 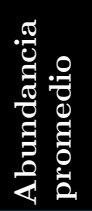 & 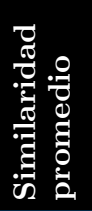 & 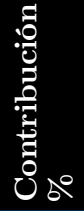 & 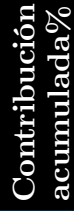 \\
\hline \multirow{5}{*}{$\begin{array}{l}\text { Pedragosa } \\
\text { Similaridad } \\
\text { promedio: } 52,5\end{array}$} & Nitzschia palea & 4,2 & 10,8 & 20,7 & 20,7 \\
\hline & Epithemia sorex & 3,7 & 8,0 & 15,3 & 35,9 \\
\hline & Ephitemia adnata & 2,3 & 4,6 & 8,7 & 44,7 \\
\hline & Nitzschia amphibia & 1,9 & 4,5 & 8,5 & 53,2 \\
\hline & Cocconeis placentula & 2,4 & 4,3 & 8,2 & 61,4 \\
\hline \multirow{5}{*}{$\begin{array}{l}\text { Mallorquina } \\
\text { Similaridad } \\
\text { promedio: } 42,8\end{array}$} & Nitzschia palea & 3,8 & 7,1 & 16,6 & 16,6 \\
\hline & Cocconeis placentula & 2,3 & 5,4 & 12,5 & 29,2 \\
\hline & Epithemia adnata & 2,0 & 4,6 & 10,8 & 40,0 \\
\hline & Navicula capitatoradiata & 1,9 & 3,3 & 7,6 & 47,6 \\
\hline & Bacillaria paxilifer & 1,8 & 3,2 & 7,6 & 55,2 \\
\hline
\end{tabular}




\begin{tabular}{|c|c|c|c|c|c|}
\hline Sitio & Especies & 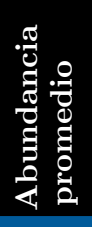 & 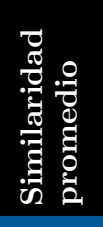 & 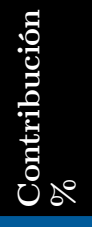 & 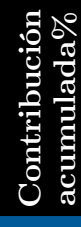 \\
\hline \multirow{5}{*}{$\begin{array}{l}\text { Sauce } \\
\text { Similaridad } \\
\text { promedio: } 39,1\end{array}$} & Cocconeis placentula & 5,5 & 23,5 & 60,1 & 60,1 \\
\hline & Gomphonema parvulum & 1,2 & 2,5 & 6,3 & 66,4 \\
\hline & Nitzschia palea & 1,4 & 2,2 & 5,7 & 72,1 \\
\hline & Nitzschia amphibia & 1,3 & 1,8 & 4,6 & 76,7 \\
\hline & Cocconeis lineata & 1,4 & 1,3 & 3,4 & 80,1 \\
\hline \multirow{7}{*}{$\begin{array}{l}\text { Salto de Agua AB } \\
\text { Similaridad } \\
\text { promedio: } 55,5\end{array}$} & Nitzschia amphibia & 3,3 & 5,8 & 10,5 & 10,5 \\
\hline & Planothidium biporomum & 3,6 & 5,6 & 10,1 & 20,6 \\
\hline & Nitzschia palea & 3,1 & 5,5 & 9,8 & 30,4 \\
\hline & Cocconeis placentula & 2,7 & 5,0 & 9,0 & 39,4 \\
\hline & Gomphonema parvulum & 2,5 & 4,7 & 8,5 & 48,0 \\
\hline & Navicula schroeterii & 2,4 & 4,3 & 7,8 & 55,8 \\
\hline & Gomphonema pumilum & 2,2 & 3,8 & 6,8 & 62,6 \\
\hline \multirow{4}{*}{$\begin{array}{l}\text { Pan de Azúcar AB } \\
\text { Similaridad } \\
\text { promedio: } 43,9\end{array}$} & Cocconeis placentula & 5,0 & 15,1 & 34,3 & 34,3 \\
\hline & Gomphonema pumilum & 2,5 & 4,8 & 11,0 & 45,2 \\
\hline & Planothidium biporomum & 1,2 & 3,4 & 7,7 & 53,0 \\
\hline & Nitzschia amphibia & 1,9 & 3,2 & 7,3 & 60,3 \\
\hline \multirow{3}{*}{$\begin{array}{l}\text { Vivero } \\
\text { Similaridad } \\
\text { promedio: } 38,7\end{array}$} & Cocconeis placentula & 4,2 & 11,6 & 29,9 & 29,9 \\
\hline & Planothidium biporomum & 3,6 & 7,0 & 18,2 & 48,1 \\
\hline & Epithemia sorex & 2,1 & 4,6 & 11,9 & 60,0 \\
\hline \multirow{5}{*}{$\begin{array}{l}\text { Represa } \\
\text { Similaridad } \\
\text { promedio: } 46,8\end{array}$} & Navicula capitatoradiata & 4,1 & 11,3 & 24,1 & 24,1 \\
\hline & Cocconeis placentula & 2,9 & 6,5 & 13,8 & 37,9 \\
\hline & Achnanthidium minutissimum & 2,6 & 4,4 & 9,3 & 47,2 \\
\hline & Planothidium biporomum & 2,2 & 3,5 & 7,5 & 54,8 \\
\hline & Encyonema sprechmanii & 1,6 & 3,2 & 6,7 & 61,5 \\
\hline
\end{tabular}

Los resultados de los valores propios, porcentaje de varianza explicada y correlaciones canónicas del CCA se muestran en la Tabla 5. El análisis de correspondencia canónico mostró que los dos primeros ejes explicaron el 48,3\% (porcentaje de varianza acumulado restringido). Las variables que más contribuyeron al primer eje fueron el PT $(\mathrm{cc}=0,721), \mathrm{pH}(\mathrm{cc}=0,485)$, y negativamente el NT $(\mathrm{cc}=-0,679)$, mientras que la conductividad $(\mathrm{cc}=0,828)$ y la turbiedad (UNT) $(\mathrm{cc}=-0,452)$ fueron los que más contribuyeron al eje 2 (Tabla 6, Figura 5). La clorofila a aportó igualmente a ambos ejes $(c c=0,54)$. Un tercer eje contribuyó con un $18 \%$ a la varianza y se explicó con el fosfato $(c c=-0,815)$. El diagrama biplot de las variables y especies considerando los dos primeros ejes mostró una correlación positiva de las especies 
al fósforo total y negativa con el nitrógeno total en el eje 1 (Figura 5). Se observó que tanto el PT como el fosfato pueden ser variables explicativas de su distribución.

La distribución de las especies en relación al NT y PT mediante las correlaciones canónicas especies-variable se representan en la Figura 6. Los puntajes de cada especie del eje 1 del CCA respecto al eje 2 muestran que las especies con correlaciones positivas son las afines al fósforo, mientras que las negativas lo son al NT.

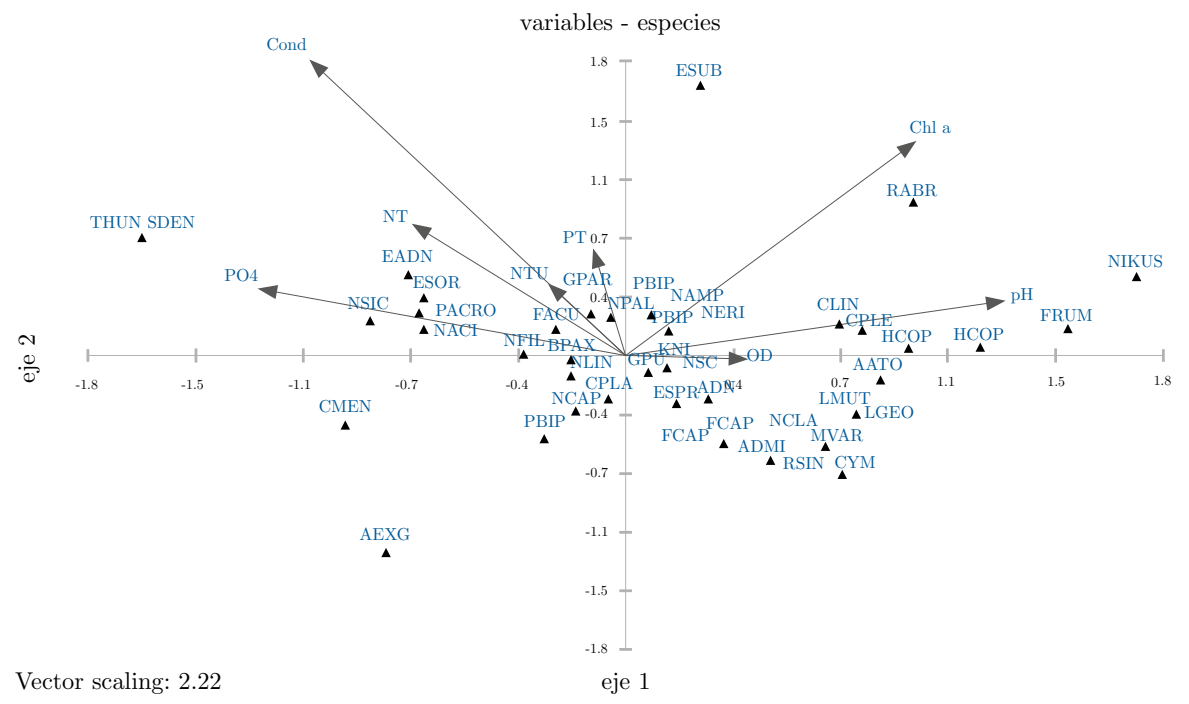

Figura 5. Análisis de correlación canónica de las especies con abundancia relativa mayor al 4\% (azul) y variables ambientales (flechas grises). Los códigos de las especies figuran en la Tabla 3 y los de las variables, en la Tabla 2.

TABLA 5. Valores propios, porcentaje de varianza explicada acumulada para el espacio no restringido y para el espacio restringido y correlaciones canónicas para los tres primeros ejes del análisis de correspondencia canónico.

\begin{tabular}{|lccc|}
\hline & Eje 1 & Eje 2 & Eje 3 \\
\hline Valores propios & 0,21 & 0,14 & 0,12 \\
\hline Porcentaje & 8,33 & 5,76 & 4,72 \\
\hline Porcentaje de varianza acumulado & 8,33 & 14,10 & 18,81 \\
\hline Porcentaje de varianza acumulado restringido & 28,61 & 48,39 & 64,58 \\
\hline Correlaciones canónicas especie-ambiente & 0,88 & 0,89 & 0,80 \\
\hline
\end{tabular}


TABLA 6. Correlaciones canónicas de las variables con los ejes.

\begin{tabular}{|lccc|}
\hline Coeficientes canónicos & Eje $\mathbf{1}$ & Eje 2 & Eje 3 \\
\hline Conductividad & $-0,41$ & $\mathbf{0 , 8 3}$ & $-0,06$ \\
\hline pH & 0,49 & 0,23 & $-1,35$ \\
\hline Turbiedad & 0,02 & $-0,45$ & 0,87 \\
\hline Oxígeno disuelto & 0,10 & 0,06 & $-0,11$ \\
\hline Nitrógeno Total & $\mathbf{- 0 , 6 8}$ & 0,10 & $-0,46$ \\
\hline Fósforo total & $\mathbf{0 , 7 2}$ & 0,02 & 0,08 \\
\hline Chl $a$ & 0,54 & 0,55 & 0,62 \\
\hline Fosfato & $-0,13$ & 0,01 & $\mathbf{- 0 , 8 2}$ \\
\hline
\end{tabular}

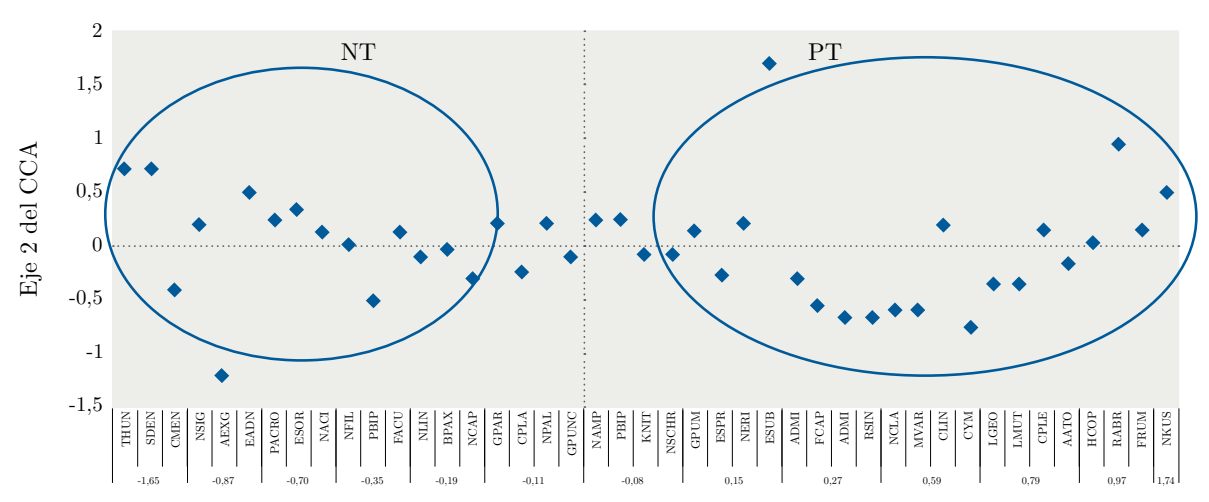

Eje 1 del CCA

Figura 6. Distribución de las especies (código en tabla 3) en el eje 1 y 2 del ACC.

Los sitios que presentaron mayor concentración de nutrientes, Pedragosa y Mallorquina, se caracterizaron por la abundancia de Nitzschia palea, Ephitemia sorex y E. adnata (Tabla 4). Estas especies se asociaron con el NT en el CCA (Figura 5 y 6). Además, en dichos sitios se registró la presencia de especies con alto Iidp como N. sigmoidea, N. acicularis y Tryblionella hungarica (Tabla 3), lo que resultó en un IDP de 2,5 y 2,6, respectivamente, correspondiente a la categoría de mala calidad del agua (Figura 7).

Los sitios Pan de Azúcar AB, Sauce y Salto de Agua AB también presentaron mala calidad del agua según su IDP y se caracterizaron por la dominancia de Cocconeis placentula, C. lineata, Gomphonema parvulum, G. pumilum, Nitzschia amphibia y Planothidium biporomum (Tabla 4). Estas especies se asociaron al PT en el eje 1 del CCA (Figura 5 y 6). A su vez, Pan de Azúcar AB presentó baja abundancia relativa $(8,3)$ de Luticola goeppertiana, especie con un alto Iidp: 3,75 .

El arroyo Enjoy estuvo caracterizado por Rhoicosphenia abbreviata y Craticula subminuscula, ambas asociadas positivamente al PT y fosfato según el eje 1 del ACC (Figura 5), pero solo Craticula subminuscula presentó alto Iidp (3,75) (Tabla 3). 
Los sitios Pan de Azúcar AA, Represa y arroyo Potrero presentaron en promedio calidad aceptable del agua; se caracterizaron por especies con bajo Iidp: Achnanthidium minutissimum y Navicula capitatoradiata (Iidp=1,25) y otras sin ponderación Iidp: Amphora pediculus, Navicula schroeterii, Encyonema sprechmanii y Planothidium biporomum (Tablas 3 y 4 ).

Los cálculos del IDP en cada sitio mostraron que la calidad de agua no varió durante el período de estudio, excepto en Pedragosa y Mallorquina. Estos sitios presentaron calidad muy mala y mala en los tres primeros muestreos (octubre de 2015 , diciembre de 2015 y abril de 2016), pero en el muestreo de setiembre de 2016 Mallorquina presentó calidad aceptable (1.5) (Figura 7).

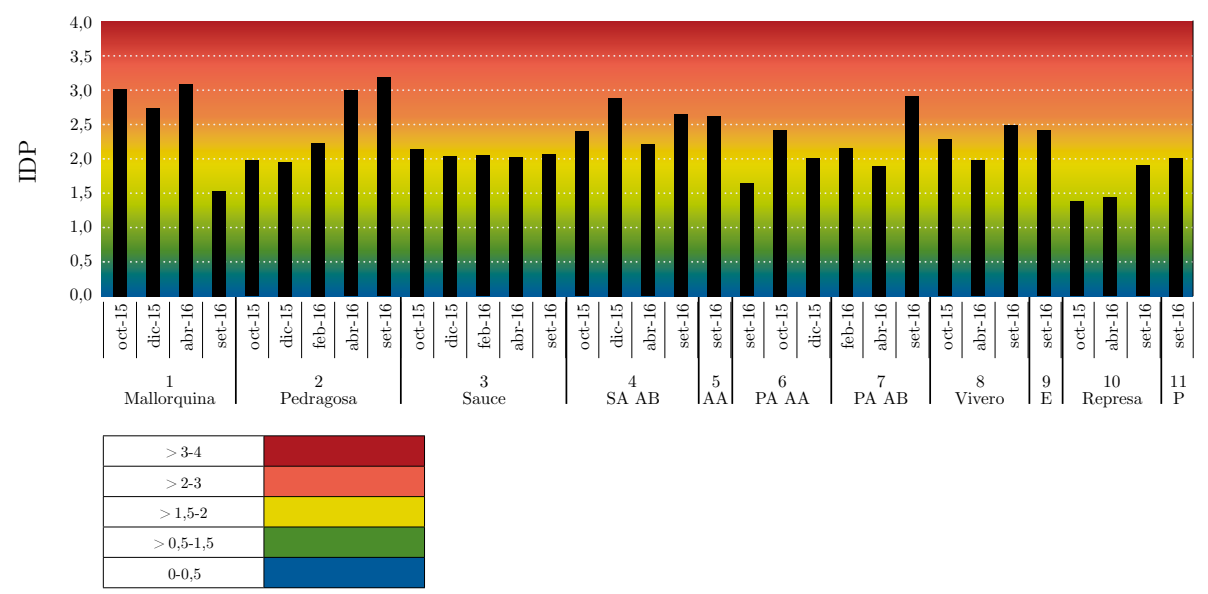

Figura 7. Valor de IDP y calidad de agua para cada sitio de muestreo durante octubre de 2015 y setiembre de 2016. Ver códigos de referencia en Tabla 1 Sitios de muestreo: (1) Mallorquina, (2) Pedregosa, (3) Sauce, (4) Salto de Agua AB, (5) Salto de Agua AA, (6) Pan de Azúcar AA, (7) Pan de Azúcar AB, (8) Vivero, (9) Enjoy, (10) Represa y (11) Potrero. Índice de colores de acuerdo a calidad de agua inferida a partir del IDP.

\section{DISCUSIÓN}

Los resultados obtenidos de este trabajo sugieren que el índice pampeano puede ser aplicado exitosamente en arroyos uruguayos. Las correlaciones entre las variables ambientales con la comunidad de diatomeas y el IDP obtenido en cada sitio reflejan las condiciones ambientales de los sistemas lóticos estudiados. El IDP reflejó a los sitios siguiendo las características ambientales obtenidas en cada muestreo.

La variación temporal del IDP dentro de un mismo sitio podría deberse a las variaciones de los usos del suelo y, por consiguiente, a la entrada de nutrientes al sistema y/o al aumento del caudal por la escorrentía y las precipitaciones en la cuenca. Los aportes de nutrientes al sistema de la laguna son numerosos y fluctúan a lo largo del año de acuerdo al uso del suelo en la cuenca. Este uso es mayoritariamente 
agropecuario, más del 50\% del área está dedicada a actividades ganaderas, y ya hace varias décadas se ha incrementado la presión por el uso recreativo en el sector sur de la Laguna, donde existen varios emprendimientos turísticos y un pequeño poblado sin saneamiento (Amorín y Larghero, 2017). Con frecuencia, los niveles altos de nitrógeno en el agua son producto del exceso de fertilizantes y en ocasiones aumentan por la erosión del suelo adyacente al cuerpo de agua, debido a sucesivos cultivos (Ferrando, 2013).

Según los resultados análisis de similitud entre sitios, también se observó una variabilidad espacial; cada arroyo estuvo determinado por una comunidad típica a través de la cual podríamos inferir cuál es el nutriente dominante.

Si bien el sistema recibe aguas de mala calidad de sus tributarios, se observó que en el sur, en el sitio Represa y sobre el arroyo Potrero hacia la salida en el Río de la Plata, la calidad del agua mejora (IDP $=1,6$ y $\mathrm{IDP}=2,0)$ y es aceptable al final del período de estudio.

Las condiciones meteorológicas influyen en la calidad del agua y, en consecuencia, en el IDP obtenido. La información meteorológica en la zona, proporcionada por el Instituto Uruguayo de Meteorología (INUMET, [s.d.]), indicó que la pluviosidad de abril a setiembre de 2016 se ubicó por encima de lo normal. La precipitación acumulada para julio de 2016 fue de $317 \mathrm{~mm}$ según la estación meteorológica de la Laguna del Sauce (García López, [s.d.]), mientras que los promedios históricos acumulados mensuales son de $93 \mathrm{~mm}$ para la estación de Carrasco (estación meteorológica más cercana que dispone datos acumulados históricos 1961-1990). Incluso previo al muestreo, el día 28/08/2016 se registraron 93 mm para La Estación de la Base Aeronaval Curbelo. Por tanto, en un día llovió lo que normalmente se espera para el mes de setiembre. Este efecto tan puntual y extraordinario, por el que el nivel de los arroyos aumentó y las compuertas de la represa del arroyo Potrero se abrieron, provocó un aumento de la corriente no observado durante el período de estudio. En consecuencia, esto podría haber provocado la mejora en la calidad del agua en Mallorquina y Pedragosa en los sitios norte, mientras que para Represa aumentó el índice y, por ende, disminuyó la calidad del agua en ese sitio.

Los tributarios de la Cuenca del Sauce no mostraron valores altos de DBO a diferencia de los arroyos pampeanos (Gómez, 1998; Licursi y Gómez, 2003). Esta diferencia podría ocurrir debido a que los arroyos argentinos son urbanos. Varios autores han demostrado la influencia de la eutrofización, la contaminación orgánica y el oxígeno disuelto como estructuradores de la comunidad de diatomeas (Bere y Tundisi, 2011; Lobo, et al., 2004a; Lobo, et al., 2010; Van Dam, et al., 1994; Prygiel y Coste, 2000), en línea con lo hallado en este estudio.

Un alto porcentaje de valvas $(70 \%)$ fueron ponderadas por el «índice específico»(Iidp), como Nitzschia, Cocconeis, Gomphonema y Navicula. Estos géneros fueron los dominantes en este estudio y concordaron con lo descripto para ambientes eutróficos y con contaminación orgánica, específicamente Gomphonema parvulum y Nitzschia palea (Bere y Tundisi, 2011; Lobo, et al., 2010; Prygiel y Coste, 2000), y para áreas de escorrentía de terrenos agrícolas o con altas concentraciones de 
nitratos y fosfatos (Alarcón-Rojas y Peláez-Peláez, 2012), como es el caso de esta zona de estudio.

El CCA ilustró la relación entre las especies indicadoras y su asociación con los dos principales nutrientes, el NT y el PT. Especies con bajo $\mathrm{I}_{\text {idp }}$ como Achnanthes exigua y Achnanthidium minutissimum se correlacionaron negativamente con el PT mientras que especies con alto $\mathrm{I}_{\mathrm{idp}}$ como Cocconeis placentula, C. lineata, Gomphonema parvulum, G. pumilum, Nitzschia amphibia, Planothidium biporomum, Rhoicosphenia abbreviata y Craticula subminuscula se asociaron a concentraciones altas de PT. Otras especies con alto $\mathrm{I}_{\mathrm{idp}}$ como N. sigmoidea, N. acicularis y Tryblionella hungarica se asociaron con altas concentraciones de NT. Pocas especies registradas no presentaban ponderación para el IDP como Planothidium biporum, generalmente dominante en varios sitios de mala calidad y asociada al PT según el CCA. Rhoicosphenia abbreviata, también carente de ponderación, es una especie dominante del sitio de mayor concentración de fósforo (Enjoy), pero contradictoriamente se reporta en la literatura como especie típica de ambiente con moderada y buena calidad del agua (Bere y Tundisi, 2011). Otro caso sin índice específico fue el de Epithemia, que en este estudio se la observó asociada a altos niveles de NT, como se tiene registro en ríos colombianos (Pedraza-Garzón y Donato-Rondón, 2011), y no donde el cociente nitrógeno/fósforo es relativamente bajo (Stancheva, et al., 2013). Este género puede tolerar bajas concentraciones de nitrato y amonio disuelto gracias a su endosimbiosis con cianobacterias que pueden fijar el nitrógeno atmosférico (EPA, et al., [s.d.]).

Este estudio da cuenta de la importancia de crear ponderaciones de especies de diatomeas aún no ponderadas que son relevantes en cuanto a su potencial como especies indicadoras de calidad del agua en los sistemas lénticos y lóticos en una región de nuestro país.

Resulta interesante seguir con los estudios en sitios donde se pueda detectar un gradiente de oxígeno y de la DBO, como arroyos urbanos de Montevideo. Asimismo sería necesario continuar con los estudios para poder clasificar a las especies que no tengan un valor de ponderación $\mathrm{I}_{\mathrm{idp}}$, ya que la cantidad de especies ponderadas puede variar el IDP y podría influenciar en la calidad resultante del agua.

Diatomeas con morfologías anormales o frústulos deformados son indicadoras de estrés ambiental y/o presencia de contaminantes no orgánicos. Futuros estudios podrían incluir el análisis de metales pesados, pesticidas y otros contaminantes para evaluar la comunidad perifítica, incorporando el porcentaje de valvas deformadas de diatomeas (Gómez y Licursi, 2003).

\section{CONCLUSIONES}

Se aplica por primera vez en Uruguay un bioindicador bentónico en monitoreos de calidad de agua en arroyos utilizando el perifiton. Este trabajo evidencia una vez más que los ensambles de diatomeas epilíticas reflejan el estado de salud del ambiente. Los programas de monitoreo de calidad de agua podrían complementarse con la 
incorporación de esta herramienta que sería de gran utilidad en el asesoramiento del estado trófico de los cuerpos de aguas lóticos.

Dar seguimiento al monitoreo de la cuenca de la Laguna del Sauce incorporando el índice diatomológico (IDP) para aportar información sobre la calidad del agua de los afluentes que llegan a la laguna permitirá anticipar y dar prevención a eventos de mala calidad de agua. Las estimaciones del estado trófico realizadas únicamente a partir de los índices fisicoquímicos dependen de las condiciones de la calidad del agua en el momento de la toma de la muestra y no necesariamente reflejan el estado ecológico real del sistema. Por lo tanto, siempre se deben analizar e interpretar los índices fisicoquímicos e indicadores ecológicos en su conjunto (Torres-Franco, et al., 2018).

Desde el punto de vista de la gestión actual, esta herramienta podría ser de utilidad para complementar la estrategia de control planteada por la Dirección Nacional de Medio Ambiente (MVOTMA, 2015) y el monitoreo que lleva adelante la OSE-UGD en colaboración con la UdelaR (Crisci, et al., 2017) para la cuenca de la Laguna del Sauce. La incorporación IDP serviría para dar seguimiento a las medidas del plan de acción que se está implementando para el aseguramiento de la potabilización del agua, no solo de este sistema estudiado, sino en otras cuencas, por ejemplo, la del Santa Lucía.

\section{RECONOCIMIENTOS}

A los colegas de OSE-UGD, JJ Lagomarsino y Gustavo Méndez por el apoyo en la ejecución e interpretación de los datos. A Nora Maidana, Departamento de Biodiversidad y Biología Experimental - FCEyN - Universidad de Buenos Aires, Argentina, quien nos recibió en sucesivas pasantías discutiendo la taxonomía de las especies. A Nora Gómez, del Instituto de Limnología Dr Raúl A. Ringuelet, de La Plata, Argentina, por el apoyo en los cálculos del IDP y por recibirnos en el instituto. A Patrick Kociolek, de University of Colorado Boulder, por el apoyo en la taxonomía. A personal de Prefectura Nacional Naval, especialmente al Cabo Sosa, de Base Aeronaval Capitán Carlos A. Curbelo de Laguna del Sauce. A los propietarios de la zona, quienes nos permitieron el acceso y la colocación de sustratos, entre ellos a Luis Hernández y Daniel Branáa. Al LATU por tener la iniciativa en llevar adelante esta temática en pos del fortalecimiento de los monitoreos de calidad de agua. A los revisores del trabajo por sus valiosas sugerencias y correcciones.

\section{REFERENCIAS}

Alarcón-Rojas, N. y Peláez-Peláez, F., 2014. Water quality of the river Sendamal (Celendin, Cajamarca, Peru): determination by use of diatoms 2012. En: Rebiol., 34(2), pp.29-37.

American Public Health Association, American Water Works Association y Water Environment Federation, 2017. Standard methods for the examination of water and wastewater. 23va ed. Washington: APHA. Standard Method 4500 NH3-D, Approved 1997-Rev. 2011. 
American Public Health Association, American Water Works Association y Water Environment Federation, 2017. Standard methods for the examination of water and wastewater. 23va ed. Washington: APHA. Standard Method 2540 D, Approved 2015.

American Public Health Association, American Water Works Association y Water Environment Federation, 2017. Standard methods for the examination of water and wastewater. 23va ed. Washington: APHA. Standard Method 4500 P, Approved 2005-Rev. 2011 and 2017.

Amorín, C. y Larghero, S., 2017. Informe de situación de las medidas que se están implementado para el aseguramiento de la potabilización del agua del sistema de abastecimiento de Montevideo y Laguna del Sauce. Montevideo: URSEA.

Asociación Española de Normalización y Certificación, 2004. UNE EN-13946: Calidad del agua. Guía para el muestreo en rutina y el pretratamiento de diatomeas bentónicas de ríos. Madrid: AENOR.

Barbour, M.T., Gerritsen, J., Snyder, B.D. y Stribling, J.B., 1999. Rapid bioassessment protocols for use in streams and wadeable rivers: periphyton, benthic macroinvertebrates and fish. 2da ed. U.S. Washington: Environmental Protection Agency, Office of Water. (EPA 841-B-99-002).

Battarbee, R.W., 1986. Diatom analysis. En: Berglund, B.E., ed., 1986. Handbook of holocene paleoecology and paleohydrology. Londres: Wiley \& Sons. pp.527-570.

Bere, T. y Tundisi, J.G., 2011. The effects of substrate type on diatom-based multivariate water quality assessment in a tropical river (Monjolinho), São Carlos, SP, Brazil. En: Water Air Soil Pollut, (216), pp.391-409.

Bere, T. y Tundisi, J.G., 2012. Applicability of the Pampean Diatom Index (PDI) to streams around São Carlos-SP, Brazil. En: Ecological Indicators, (861), pp.5.

Blanco Lanza, S., Álvarez Blanco, I., Cejudo-Figueiras, C. y Bécares Mantecón, E. 2010. Guía de las diatomeas de la Cuenca del Duero. Madrid: Confederación Hidrográfica del Duero. Ministerio Medio Ambiente de España. 210p.

Cambra, J., Luc, Héctor y Sabater, S., 2005. Métodos para el establecimiento el estado ecológico según la Directiva Marco del Agua. Protocolos de muestreo y análisis para fitobentos [En línea]. Madrid: Confederación Hidrográfica del Ebro. Ministerio de Medio Ambiente de España. 43 p. [Consulta: 17 de mayo de 2019]. Disponible en: http://195.55.247.234/webcalidad/estudios/indicadoresbiologicos/ Manual_fitobentos.pdf

Carpenter, S., Stanley, E.H. y Vander Zanden, M.J., 2011. State of the world's freshwater ecosystems: physical, chemical, and biological changes. En: Annual Review of Environment and Resources, (36)1, pp.75-99.

Cemagref, 1982. Etude des méthodes biologiques d'appréciation quantitative de la qualité des eaux. Lyon: Agence de l'Eau Rhône-Méditerranée-Corse. p. 218.

Clarke, K.R. y Warwick, RM., 2001. Change in marine communities: an approach to statistical analysis and interpretation. 2a ed. Plymouth: PRIMER-E.

Clarke, K.R. y Gorley R.N., 2006. PRIMER v6: User Manual/Tutorial. Plymouth: Plymouth Marine Laboratory. 190 pp. 
Confederación Hidrográfica del Ebro, 2007. Metodología para el establecimiento del estado ecológico según la Directiva del Marco del Agua en la Confederación Hidrográfica del Ebro: Protocolos de muestreo y análisis para: Fitoplancton, Fitobentos (Microalgas bentónicas), Macrofitos, Invertebrados bentónicos, Ictiofauna [En línea]. Madrid: Ministerio de Medio Ambiente. [Consulta: 17 de mayo de 2019]. Disponible en: https://www.miteco.gob.es/es/agua/publicaciones/ Protocolos_muestreo_biologico_con_portada_tcm30-214764.pdf

Coste, M. y Ayphassorho, H., 1991. Étude de la qualité des eaux du Bassin Artois Picardie a l`aide des communautés de diatomées benthiques (Application des indices diatomiques). Douai: Agence de l`Eau Artois-Picardie. (Report Cemagref. Bordeaux). 227 pp.

Coste, M., Boutry, S., Tison-Rosebery, J. y Delmas, F., 2008. Improvements of the Biological Diatom Index (BDI): description andefficiency of the new version (BDI2006). En: Ecol. Indic., 9, pp.621-650. DOI 10.1016/j.ecolind.2008.06.003.

Crisci, C., Goyenola, G.,Terra, R., Lagomarsino, J.J., Pacheco, J.P., Díaz, I., Gonzalez-Madina, L., Levrini, P., Méndez, G., Bidegain, M., Ghattas, B.A. y Mazzeo, N.R., 2017. Dinámica ecosistémica y calidad de agua: estrategias de monitoreo para la gestión de servicios asociados a Laguna del Sauce (Maldonado, Uruguay). En: INNOTEC, 13, pp.46-57. ISSN 1688-6593.

Descy, J.P. y Coste, M., 1991. A test of methods for assesing water quality based on diatoms. En: Verh. Internat. Verein. Limnol., Stuttgart, (24), pp.2112-2116.

Duarte, C., Sergio A., Benito, G., Dachs, J., Montes, C., Pardo, M., Ríos, A.F., Simóy, R. y Valladares, F., 2006. Cambio global Impacto de la actividad humana sobre el sistema Tierra. Madrid: Consejo Superior De Investigaciones Científicas. 170 p. (Colección Divulgación). ISBN: 978-84-00-08452-3

EPA, USGS e INSTAAR, [s.d.]. Diatoms of North America [En línea]. [s.l.]: EPA, USGS, INSTAAR. [Consulta: 1 de setiembre de 2017]. Disponible en: https://westerndiatoms.colorado.edu.

Ector, L. y Rimet, F., 2005. Using bioindicators to assess rivers in Europe: An overview. En: Lek, S., Scardi, M., Verdonschot, P. F. M., Descy, J. P. y Park, Y. S., eds. Modelling community structure in freshwater ecosystems. Berlin: Springer Verlag, Heidelberg. pp.7-19.

Ferrando M., 2013. Curso de fertilidad de suelos [En línea]. Montevideo: Facultad de Agronomía. [Consulta: 1 de setiembre de 2017]. Disponible en: http://www.fagro. edu.uy/fertilidad/curso/docs/Fosforo.pdf

García López, José Manuel, [s.d.]. OGIMET, Servicio de Información Meteorológica. [s.l.]: [s.n.]. [Consulta: 17 de mayo de 2019]. Disponible en: http://ogimet.com/cgibin $/$ gsynres $?$ ind $=86586 \&$ ndays $=30 \& a n o=2016 \&$ mes $=05 \&$ day

$=01 \&$ hora $=12 \&$ ord $=$ DIR\&enviar $=$ Ver

Gómez, N., 1998. Use of epipelic diatom for evaluation of water quality in the Matanza-Riachuelo (Argentina), a pampean plain river. En: Water Research, 32, pp.2029-2034. 
Gómez, M. y Licursi, M., 2003. Abnormal forms in Pinnularia gibba (Bacillariophyceae) in a polluted lowland stream from Argentina. En: Nova Hedwigia, 77(3), pp.389-398.

Gómez, N. y Licursi, M., 2001. The Pampean Diatom Index (IDP) for assessment of rivers and streams in Argentina. En: Aquatic Ecol., 5, pp.173-181.

González-Madina, L., Pacheco, J. P., Mazzeo, N., Levrini, P., Clemente, J., Lagomarsino J.J. y Fosalba, C., 2017. Factores ambientales controladores del fitoplancton con énfasis en las cianobacterias potencialmente tóxicas en un lago somero utilizado como fuente de agua para potabilización: Laguna del Sauce, Maldonado, Uruguay. En: INNOTEC, 13, pp.26-35. ISSN 1688-6593.

Hill, B., Herlihy, A., Kaufmann, P., Stevenson, R., McCormick, F. y Johnson, C., 2000. Use of periphyton assemblage data as an index of biotic integrity. En: Journal of the North American Benthological Society, 19, pp.50-67.

Hill, B., Stevenson, R., Pan, Y., Herlihy, A., Kaufmann, P. y Jhonson C., 2001. Comparison of correlations between environmental characteristics and stream diatom assemblages characterized at genus and species levels. En: Journal of the North American Benthological Society, 20, pp.299-231.

International Organization for Standardization, 1992. ISO 10260: Water quality -- Measurement of biochemical parameters -- Spectrometric determination of the chlorophyll-a concentration. Ginebra: ISO .

International Organization for Standardization, 1997. ISO 11905-2: Water quality -- Determination of nitrogen -- Part 2: Determination of bound nitrogen, after combustion and oxidation to nitrogen dioxide, chemiluminescence. Ginebra: ISO .

International Organization for Standardization, 1999. ISO 8245: Water quality

-- Guidelines for the determination of total organic carbon (TOC) and dissolved organic carbon (DOC). Ginebra: ISO .

International Organization for Standardization, 2002. ISO 15705: Water quality

-- Determination of the chemical oxygen demand index (ST-COD) -- Small-scale sealed-tube method. Ginebra: ISO .

International Organization for Standardization, 2003. ISO 5815-2: Water quality -- Determination of biochemical oxygen demand after $n$ days (BODn) -- Part 2: Method for undiluted samples. Ginebra: ISO .

International Organization for Standardization, 2007. ISO 10304-1: Water quality

-- Determination of dissolved anions by liquid chromatography of ions -- Part 1:

Determination of bromide, chloride, fluoride, nitrate, nitrite, phosphate and sulfate. Ginebra: ISO .

INUMET, [s.d.]. Recursos hídricos [En línea]. Montevideo: INUMET. [Consulta: 1 de setiembre de 2017]. Disponible en: http://www.meteorologia.com.uy/ServCli/ipe https://www.inumet.gub.uy/clima/estadisticas-climatologicas/graficasestadisticas-pluviometricas

Kelly, M.G. y Whitton, B.A., 1995. The trophic diatom index: a new index for monitoring eutrophication in rivers. En: Journal of Applied Phycology, (7), pp.433-444. 
Kelly, M.G., Cazaubon, A., Coring, E., Dell'Uomo, A., Ector, L., Goldsmith, B., Guasch, H., Hurlimann, J., Jarlman, A., Kawecka, B., Kwandrans, J., Laugaste, R., Lindstrom, E.A., Leitao, M., Marvan, P., Padisaka, J., Pipp, E., Prygiel, J., Rott, E., Sabater, S., van Dam, H. y Vizinet J., 1998. Recommendations for the routine sampling of diatoms for water quality assessments in Europe. En: Journal of Applied Phycology, 10, pp.215-224.

Krammer, K., 2000. Diatoms of Europe: the Genus Pinnularia. Vol. 1. Ruggell: A.R.G. Gantner Verlag K.G.

Krammer, K., 2002. Diatoms of Europe: Cymbella. Vol. 3. Ruggell: A.R.G. Gantner Verlag K.G.

Krammer, K., 2003. Diatoms of Europe : Cymbopleura, Delicata, Navicymbula, Gomphocymbellopsis, Afrocymbella. Vol. 4. Ruggell: A.R.G. Gantner Verlag K.G.

Lachat Instruments, 2007. QuikChem(R 31-115-01-3-D. Milwaukee: Lachat Instruments

Lange-Bertalot, H. 2001. Diatoms of Europe: Navicula sensu stricto 10 genera separated from Navicula sensu lato Frustulia.Vol. 2. Ruggell: A.R.G. Gantner Verlag K.G.

Lange-Bertalot H., Bak, M., Witkowski A. y Tagliaventi N., 2011. Diatoms of Europe: Eunotia and some related genera. Vol. 6. Ruggell: A.R.G. Gantner Verlag K.G.

LeCointe, C., Coste, M. y Prygiel, J., 1993. «OMNIDIA»software for taxonomy, calculation of diatom indices and inventories management. En: Hydrobiologia, 269/270, pp.509-513.

Legendre, P. y Legendre, L., 2012. Numerical ecology. 3ra ed. Amsterdam: Elsevier. 1006p.

Lenoir, A. y Coste, M., 1996. Development of a practical diatom index of overall water quality applicable to the French national water Board network. En: Whitton, B. y Rott, E., eds. Use of algae for monitoring rivers. Vol. 2. Innsbruck: STUDIA, Studenten-förderungs-Ges. m.b.H.

Levkov, Z., 2009. Diatoms of Europe: Amphora sensu lato. Vol. 5. Ruggell: A.R.G. Gantner Verlag K.G.

Levkov, Z., Metzeltin, D. y Pavlov, A., 2013. Diatoms of Europe: Luticola and Luticolopsis. Vol. 7. Ruggell: A.R.G. Gantner Verlag K.G.

Levkov, Z., Mitic, D., Kopanja y Reichardt, E., 2016. Diatoms of Europe: The diatom genus Gomphonema from the Republic of Macedonia. Vol. 8. Ruggell: A.R.G. Gantner Verlag K.G.

Li, L., Zheng, B. y Liu, L., 2010. Biomonitoring and bioindicators used for river ecosystems: definitions, approaches and trends. En: Procedia Environmental Sciences, 2, pp.1510-1524.

Licursi, M. y Gómez, N., 2003. Aplicación de índices bióticos en la evaluación de la calidad del agua en sistemas lóticos de la llanura Pampeana argentina a partir del empleo de diatomeas. En: Biología Acuática, 21, pp.31-49.

Lobo, E. A. y Callegaro, V. L., 2000. Avaliação da qualidade de águas doces continentais com base em algas diatomáceas epilíticas: enfoque metodológico. 
En: Tucci, C.E.M. y Marques, D.M., eds., 2000. Avaliação e controle da drenagem urbana. Porto Alegre: Ed. Universidade/UFRGS. pp. 277-300.

Lobo, E.A., Callegaro, V.L., Hermany, G., Bes, D., Wetzel, C.E. y Oliveira, M.A., 2004a. Use of epilithic diatoms as bioindicator from lotic systems in Southern Brazil, with special emphasis in eutrophication. En: Acta Limnol, Brasil., 16, pp.25-40.

Lobo, E., Callegaro, V., Hermany, G. y Ector, L., 2004b. Review of the use of microalgae in South America for monitoring rivers, with special reference to diatoms. En: Vie et Milieu, (53), pp.35-45.

Lobo, E. A., Wetzel, C .E., Ector, L., Katoh, K., Blanco, S. y Mayama, S., 2010. Response of epillithic diatom communities to environmental gradients in subtropical temperate Brazilian Rivers. En: Limnetica., 29(2), pp.323-340.

Mazzeo, N., García-Rodríguez, F., Rodríguez, A., Méndez, G., Iglesias, C., Inda, H., Goyenola, G., García, S., Fosalba, C., Marroni, S., Crisci, C., Del Puerto, L., Clemente, J., Pacheco, P., Carballo, C., Kröger, A., Vianna, M., Meerhoff, M., Steffen, M., Lagomarsino, J.J., Masdeu, M., Vidal, N., Texeira de Mello, F., González, I. y Larrea, D., 2010. Estado trófico de Laguna del Sauce y respuestas asociadas. En: Inda, H. y Steffen, M., eds. Bases técnicas para el manejo integrado de Laguna del Sauce y cuenca asociada. Montevideo: Editorial Montevideo. pp.3149. ISBN: 978-9974-0-0694-2.

Méndez, G., Lagomarsino, J.J. y Alcorta, R., 2010. Mitigación de las consecuencias adversas en el proceso de potabilización. En: Inda, H. y Steffen, M., eds. Bases técnicas para el manejo integrado de Laguna del Sauce y cuenca asociada. Montevideo: Editorial Montevideo. pp.31-49. ISBN: 978-9974-0-0694-2.

Metzeltin, D., Lange-Bertalot. H. y García-Rodríguez, F., 2005. Diatoms of Uruguay taxonomy, biogeography, diversity. Koenigstein: Gantner Verlag.

MVOTMA, 2015. Plan de acción para la protección de la calidad ambiental y disponibilidad de las fuentes de agua potable [En línea]. Montevideo: MVOTMA. [Consulta 24 de octubre de 2017]. Disponible en: http://www.mvotma.gub.uy/ images/PLAN_DE_ACCION_Cuenca_Laguna_del_Sauce_junio_de_2015.pdf

Pedraza-Garzón, E. y Donato-Rondón, J., 2011. Diversidad y distribución de diatomeas en un arroyo de montaña de los andes colombianos. En: Caldasia, 33(1), pp.177-191.

Prygiel, J. y Coste, M., 2000. Guide metodologique pour la mise en oeuvre de l'Indice Biologique Diatomées: NF T90-354. [s.1.]: Cemagref -Groupement de Bordeaux. Water Agencies.

Prygiel, J., Whitton, B. y Bukowska, J., eds., 1999. Use of algae for monitoring rivers III. Douai: Agence de l'Eau Artois-Picardie.

Rodríguez, A., Méndez, G., Inda, H., Lagomarsino, J.J. y Steffen, M., 2010. Características y problemática de la Laguna del Sauce. En: Inda, H. y Steffen, M., eds., 2010. Bases técnicas para el manejo integrado de Laguna del Sauce y cuenca asociada. Montevideo: Editorial Montevideo. pp.14-17. ISBN: 978-9974-0-0694-2.

Sar, E.A., Sala, S.E., Sunesen, I., Henninger, M. S. y Montastruc, M., 2009. Catálogo de los géneros, especies y taxa infraespecíficos erigidos por J. Frenguelli. [s.l.]: ARG Gunter Verlag KG. (Diatom Monograph, 10). 
Sládecék, V., 1986. Diatoms as indicators of organic pollution. En: Acta Hydrochimica et Hydrobiologica, Dresden, 14(5), pp.555-566.

Springer, M., 2010. Biomonitoreo acuático. En: Revista de Biología Tropical, 58(4), pp.53-59.

Stancheva, R., Sheath, R.G., Read, B.A., McArthur, K.D., Schroepfer, C., Kociolek, J.P. y Fetscher A.E., 2013. Nitrogen-fixing cyanobacteria (free-living and diatom endosymbionts): their use in southern California stream bioassessment. En: Hydrobiologia, (720), pp.111-127.

ter Braak, C.J., 1986. Canonical correspondence analysis: a new eigenvector technique for multivariate direct gradient analysis. En: Ecology, (67), pp.1167-1169.

Torres-Franco, A.F., Alatrista-Góngora, G.F., Guzmán-Rodríguez, N.P., CalizayaAnco, J.A., Mota, C.R. y Figueredo, CC., 2018. Physicochemical and diatom trophic indexes:A complementary approch for improving wáter sustainability in a high Andean urban stream. En: Ecohydrol. Hydrobiology. htts://doi.org/10.1016/j. ecohyd.2019.01.007

Unión Europea. Directiva 2000/60/CE del Parlamento Europeo y el Consejo de 23 de octubre de 2000 por la que se establece un marco comunitario de actuación en el ámbito de la política de aguas. Diario Oficial de la Comunidad Europea, 22 de diciembre de 2000, L 327, pp.72.

Uruguay. Decreto 253/979, de 9 de mayo de 1979. Diario Oficial, 31 de mayo de 1979, No. 20.504, p.1473.

Van Dam, H., Mertens, A. y Sinkeldam, J., 1994. A coded checklist and ecological indicator values of freshwater diatoms from The Netherlands. En: Netherlands Journal of Aquatic Ecology, 28, pp.117.

Watanabe, T., Asai, K. y Houki A., 1986. Numerical estimation to organic pollution of flowing water by using epilithic Diatom Assemblage Index (DAIPo). En: The Science of total Environmental, (55), pp.209-218. 